\title{
Consecuencias no intencionales y figuración: una incursión crítica en la obra de Norbert Elias
}

\author{
Sofía Gaspar ${ }^{1}$ \\ Universidad Complutense de Madrid
}

RESUMEN

El objetivo de este artículo es analizar la centralidad teórica de un tema clásico en sociología —las «consecuencias no intencionales de la acción» (CNI) - a través de una incursión en la obra de Norbert Elias. En primer lugar, se procura delimitar los presupuestos analíticos subyacentes a las CNI, teniendo como base la revisión crítica de Ramón Ramos, quien procura revitalizar su carácter operativo en la sociología contemporánea. A continuación, se analizan en dos apartados, en articulación con la propuesta de Ramos, el modo como Elias emprende en su proyecto académico la tarea de orientar el objeto de la sociología hacia la superación de ciertos dualismos tradicionales —individuo/sociedad, acción/estructura, micro/macro—, introduciendo para tal el concepto de figuración en el centro de su análisis, como promesa de síntesis operativa en la teoría social. Este término integra en sí mismo dos niveles analíticos frecuentemente diferenciados uno del otro: estructuras intencionales y no intencionales. Para Elias, en el proceso de figuración existen: a) interdependencias no intencionales entre acciones intencionales; b) prevaleciendo estas interdependencias no intencionales sobre las acciones intencionales de los individuos cuando se analizan los procesos sociales. Así pues, intenciones e individuos son constituidos por interdependencias no intencionales en el proceso de figuración. Para finalizar, se cuestionan las potencialidades y limitaciones teóricas de la obra de Elias, y su persistente tentativa en distanciarse tanto del holismo como del individualismo metodológico.

Palabras clave: Teoría Sociológica, Teoría de la acción, Norbert Elias.

1 Agradezco al Profesor Emilio Lamo de Espinosa y a Fernando Ampudia de Haro los comentarios críticos y observaciones acerca de este trabajo. Sus sugerencias han sido imprescindibles para poder desarrollar este artículo. 


\section{LAS CONSECUENCIAS NO INTENCIONALES DE LA ACCIÓN COMO UNO DE LOS PROBLEMAS CENTRALES EN LA SOCIOLOGÍA}

La importancia de las consecuencias no intencionales de la acción, como uno de los elementos centrales y constitutivos de la teoría sociológica, es un tema largamente debatido a lo largo de la historia de esta ciencia. Aunque esta cuestión haya sido tratada bajo terminología sociológica distinta, Merton ${ }^{2}$ glosa algunas de sus denominaciones presentes en la literatura - providencia, fuerzas sociales, paradojas sociales, heterogeneidad de fines, causalidad inmanente, principio de emergencia-, así como se refiere a la diversidad de contextos relacionados con las consecuencias imprevistas - problema del mal, responsabilidad moral, libre albedrío, predestinación, fatalismo, comportamiento lógico o ilógico, predicción y control social.

El análisis de las intenciones de nuestras acciones y sus consecuencias no deseadas o previstas permite entender cómo funcionan las sociedades. Probablemente, muchos de los hechos históricos son «no intencionales». Pero, en realidad, la mayoría de las formas de vida continuas o cambiantes tienen que ser interpretadas como una mezcla de consecuencias intencionadas (reproducción social) y no intencionadas (cambio social).

En un artículo donde procura redefinir y tematizar la centralidad de las paradojas pragmáticas en la ciencia social, Ramón Ramos ${ }^{3}$ hace viable una distinción entre las «profecías reflexivas» y las «consecuencias no intencionales de la acción intencional», con la intención de integrarlas en el estudio de las paradojas sociales. Analicemos así las características inherentes a ambas, para posteriormente delimitar sus rasgos diferenciales como integrantes del objeto de la sociología.

Las profecías reflexivas, formalizadas por primera vez por Robert K. Merton, han sido analizadas por varios teóricos, que, partiendo de la definición inicial mertoniana, la han extendido a niveles explicativos de la realidad social más integrados. De entre ellos, merece la pena destacar D. Krishna, R. L. Henshel, A. Giddens, E. Lamo de Espinosa, y N. Luhmann y R. De Giorgi ${ }^{4}$. Merton parte del conocido y ampliamente citado teorema de W. I. Thomas

2 R. K. Merton, Ambivalencia sociológica y otros ensayos, Madrid, Espasa-Calpe, 1980.

3 R. Ramos Torre, «Una aproximación a las paradojas de la acción social», en E. Lamo de Espinosa y J. E. Rodríguez lbáñez (eds.), Problemas de la teoría social contemporánea, Madrid, Centro de Investigaciones Sociológicas, 1994, pp. 435-471.

4 Véase D. Krishna, "The self-fulfilling prophecy" and the nature of society», American Sociological Review, 36, 1971, pp. 1104-1107; R. L. Henshel, «The boundary of the self-fulfilling prophecy and the dilemma of social prediction», The British Journal of Sociology, 33, 4, 1982, pp. 511-528; A. Giddens, The Constitution of Society, Oxford, Polity Press, 1984; E. Lamo de Espinosa, La sociedad reflexiva, Madrid, Centro de Investigaciones Sociológicas, 1990; N. Luhmann y R. De Giorgi, Teoria della società, Milano, Franco Angeli, 1992. 
— «si los individuos definen las situaciones como reales, éstas son reales en sus consecuencias»5-, para acordar la importancia que el tema ha tenido en las obras del obispo Bossuet, Mandeville, Marx y Freud.

Ahora bien, Merton interpreta y concretiza el teorema señalando que «las definiciones públicas de una situación (profecías o predicciones) llegan a ser parte integrante de la situación y, en consecuencia, afectan a los acontecimientos posteriores» ${ }^{6}$. El autor opera aquí una distinción entre las Profecías que se Auto-Cumplen (i.e., de una definición falsa de la situación ocurre una acción nueva, que hace verdadero lo que inicialmente se suponía falso) y las Profecías que se Auto-Niegan (i.e., predicciones futuras de acontecimientos sociales que, cuando se tornan públicas, conducen a una nueva acción que cambia la situación originalmente definida).

Detengámonos ahora un momento: la definición inicial de la situación no es, en este caso, irreflexiva, espontánea o inmediata; al contrario, presupone una creencia consciente y racional (aunque posiblemente falsa) ante la situación. Estamos, así pues, describiendo una definición reflexiva de la situación, que nos lleva a una determinada consecuencia. Lo que interviene claramente en el caso de las profecías reflexivas es la presencia de un elemento central - la acción reflexiva - que media la creencia o expectativa inicial y su resultado futuro ${ }^{7}$. Ésta es una idea central en la sistematización crítica elaborada por Ramos, para quien «el producto (la consecuencia) precisa del productor (la acción), pero se desentiende de sus designios (intención)»8.

Lo que nos interesa ahora es qué tipo de resultados o consecuencias se derivan de esa acción reflexiva. O bien el producto de la acción es coincidente con la intención inicial, o bien produce resultados distintos, más allá de los previstos originalmente. Si estamos en presencia de este último caso (que es seguramente mucho más común de lo que suponemos), nos encontramos con una consecuencia no intencional de una acción intencional. Conviene, con todo, subrayar aquí que las CNI pueden igualmente derivar de una acción no intencional (p.e., acciones rutinarias cotidianas que no implican reflexividad en su ejecución) ${ }^{9}$, aunque lo que nos interesa observar en el marco analítico de este trabajo sean las

5 R. K. Merton, Teoría y estructura sociales, México, FCE, 1995, p. 505.

6 R. K. Merton, op. cit., pp. 506-507.

7 Sobre los varios tipos de acciones reflexivas - acción reflexiva (auto)consciente, autorreferente y constitutiva一, véase R. Ramos Torre, op. cit., pp. 439-446.

8 R. Ramos Torre, op. cit., p. 451.

9 Sobre este tema, veánse R. Ramos Torre, op. cit., y G. H. von Wright, Norm and Action, London, The Humanities Press, 1963. 
CNI de una acción intencional. Por otro lado $-\mathrm{y}$ éste es uno de los elementos que distingue las CNI de las profecías reflexivas-, hay que diferenciar entre las intenciones que preceden la acción de la proyección futura de lo que va a ocurrir. Una y otra no tienen por qué ser coincidentes, aunque estén indudablemente relacionadas.

Efectivamente, el tema de las CNI de la acción intencional ha sido ampliamente identificado por varios autores que, aunque apoyados en matices teóricos distintos, han destacado su relevancia analítica para el entendimiento de la naturaleza social. En el ámbito de la filosofía, la cuestión ha sido tratada por Kant, Hegel, y en la sociología clásica, por Marx, Tocqueville, Weber, Simmel y Parsons ${ }^{10}$. En paradigmas teóricos más recientes, el tema aparece problematizado en la teoría organicista de Von Hayek, en el estructural-funcionalismo de Merton, en el enfoque de la elección racional de Boudon y Elster, en la teoría de las figuraciones de Elias, en Hirschmann, Lamo de Espinosa y, de forma esquemática, en Ramos ${ }^{11}$.

En su intento de delimitar las consecuencias no intencionales, Baert ${ }^{12}$ refiere cuatro dificultades analíticas subyacentes a su tematización: 1) la existencia previa de intencionalidad y reflexividad en la acción original; 2) el hecho de que cada acción intencional conduce a un número infinito de consecuencias, de modo que es fundamental clarificar cuáles son aquellas que poseen importancia sociológica; 3) el problema en diferenciar entre la causa y el efecto de una acción dada; 4) la existencia de varios casos en que no sólo una sino varias intenciones están subyacentes al inicio de una acción.

En este contexto, procuraremos de seguido centralizar la propuesta de Ramos ${ }^{13}$, quien, apoyándose explícitamente en las obras de Merton $^{14}$, de Boudon ${ }^{15}$ y de Baert ${ }^{16}$, intenta

10 Para un repaso del modo cómo este tema se encuentra situado en un nivel macrosociológico en las obras de estos autores, véase R. Boudon, La lógica de lo social. Introducción al análisis sociológico, Madrid, Ediciones Rialp, 1981.

11 F. A. von Hayek, New Studies in Philosophy, Politics and the History of Ideas, London, Routledge \& Kegan Paul, $1978 ;$ F. A. von Hayek, Studies in Philosophy, Politics and Economics, London, Routledge \& Kegan Paul, 1967; R. K. Merton, Teoría y estructura sociales, México, FCE, 1995; R. K. Merton, Ambivalencia sociológica y otros ensayos, Madrid, Espasa-Calpe, 1980; R. Boudon, La lógica de lo social. Introducción al análisis sociológico, Madrid, Ediciones Rialp, 1981; J. Elster, El cambio tecnológico, Barcelona, Gedisa, 1990; N. Elias, Sociología fundamental, Barcelona, Gedisa, 2. a ed, 1999; A. Hirschman, «Deux cents ans de rhétorique réactionnaire: le cas de l'effect pervers", Annales ESC, 1, 1989, pp. 67-86; A. Hirschman, Las pasiones y los intereses, México, FCE, 1978; E. Lamo de Espinosa, La sociedad reflexiva, Madrid, Centro de Investigaciones Sociológicas, 1990; y R. Ramos Torre, «Una aproximación a las paradojas de la acción social», en E. Lamo de Espinosa y J. E. Rodríguez lbáñez (eds.), Problemas de la teoría social contemporánea, Madrid, Centro de Investigaciones Sociológicas, 1994, pp. 435-471.

12 P. Baert, «Unintended consequences: a typology and examples», International Sociology, 6, 2, 1991, pp. 201-210.

13 R. Ramos Torre, op. cit.

14 R. K. Merton, Ambivalencia sociológica..., op. cit.

15 R. Boudon, op. cit.

16 P. Baert, op. cit. 
construir un mapa teórico operativo que posibilite una integración crítica de este tema en la teoría sociológica de la acción.

En la explicación que desarrollan de las consecuencias no intencionales de las acciones intencionales, cualquiera de estos autores (contrariamente, como veremos más adelante, a Norbert Elias) parte de una distinción básica en la acción intencional entre los elementos volitivos o emocionales (lo que se desea o quiere que ocurra) y los cognitivos (lo que se prevé o espera que ocurra $)^{17}$. Con base a esta distinción, y apoyándose en la propuesta de Boudon ${ }^{18}$, Ramos ${ }^{19}$ emprende una especificación de los casos en que las consecuencias son: a) previstas y queridas; b) no previstas y queridas; c) previstas y no queridas; d) ni previstas ni queridas. Este último caso sería el más interesante para el análisis sociológico, toda vez que los elementos volitivos y racionales constitutivos de la propia acción no son materializados en sus consecuencias.

CUADRO 1

Consecuencias intencionales y no intencionales. En R. Ramos Torres (1994: 449)

Previsión

$(+)$

$(-)$

\begin{tabular}{llll}
\hline Propósito & $(+)$ & 1.a) Prevista y querida & 1.b) No prevista y querida \\
\cline { 2 - 4 } & $(-)$ & 1.c) Prevista y no querida & 1.d) No prevista y no querida
\end{tabular}

Ramos continúa su propuesta alegando que, para el caso de las consecuencias no previstas ni queridas, puede suceder que haya situaciones de sobreintencionalidad — cuando las $\mathrm{CNI}$ se agregan a las intenciones-y de contraintencionalidad — cuando las CNI sustituyen o desplazan las intenciones de los actores_-20. Como se observa en el cuadro 2 , se producen

17 Aunque Merton (1980), Boudon (1981) y Ramos (1994) partan de un punto de vista epistemológico que se centra en el análisis que tiene para el actor las consecuencias no intencionales, Baert (1991) extiende su reflexión a otros niveles más amplios que incluyen: 1.a) consecuencias individuales para el actor que ha desempeñado la acción; 1.b) consecuencias individuales para otros actores, distintos de aquel que ha desempeñado la acción; 1.c) consecuencias sociales, que se diferencian en: 1.c.i) consecuencias sistémicas referentes al funcionamiento/disfuncionamiento del sistema social, traducibles en problemas de cohesión y solidaridad; 1.c.ii) consecuencias agregadas que pueden motivar otras acciones individuales.

18 R. Boudon, op. cit.
19 R. Ramos Torre, op. cit.
20 Estos dos conceptos han sido inicialmente definidos por J. Elster, Logic and Society, Chichester, J. Wiley \& Sons, 1978. 
aquí otros cuatro casos en que: a) las consecuencias no intencionales se agregan a las esperadas y deseadas; b) desplazan las esperadas y se agregan a las deseadas; c) se agregan a las esperadas y desplazan las deseadas; d) las que desplazan bien las esperadas, bien las deseadas. También aquí, el caso d) —representativo de las consecuencias progresivamente contraintencionales - es el que más atrae la problematización de la comunidad sociológica.

\section{CUADRO 2}

Consecuencias sobreintencionales y contraintencionales. En R. Ramos Torres (1994: 449)

Consecuencia no intencional

\begin{tabular}{lllr} 
& & Se agrega a lo previsto & Desplaza a lo previsto \\
\cline { 3 - 4 } Consecuencia no intencional & Se agrega a lo querido & 2.a) & 2.b) \\
\cline { 2 - 4 } & Desplaza a lo querido & 2.c) & 2.d)
\end{tabular}

Por último, y con base a la distinción de Boudon ${ }^{21}$ de «lo deseado/previsto y lo deseable/previsible ${ }^{22}$ —donde el primer par se refiere al punto de vista del actor que ejecuta la acción, mientras que el segundo alude al punto de vista de un observador externo-, Ramos opera una distinción entre aquellos casos en que: a) aunque no sean esperadas ni deseadas, las consecuencias no intencionales son previsibles y deseables; $b$ ) en que no son previsibles, pero sí deseables; $c$ ) en que son previsibles, pero no deseables; d) y cuando ni son previsibles ni deseables. De igual modo, este último ejemplo evidencia el caso más importante y central en la mirada sociológica.

CUADRO 3

Deseabilidad y previsibilidad de las consecuencias no intencionales.

En R. Ramos Torre (1994: 450)

Previsibilidad

$(+)$

Deseabilidad

(+) 3.a) Previsible y deseable

3.b) Imprevisible y deseable

(-) 3.c) Previsible e indeseable

3.d) Imprevisible e indeseable

221 R. Boudon, op. cit.

22 Igualmente referida por Baert (1991), para quien las consecuencias no intencionales no tienen por qué ser indeseables y no previamente contempladas. 
Con esto, Ramos construye un dispositivo operatorio que permite observar un nivel gradativo de la importancia de las consecuencias no intencionales; los casos más centrales e interesantes en sociología son todos aquellos designados mediante la le$\operatorname{tra} d)$.

Conviene señalar aquí una vez más que las consecuencias no intencionales de la acción remiten, obviamente, a la acción intencional a lo largo de este proceso. Ramos distingue cuatro elementos subyacentes a su estructura, con el objetivo de relacionarlos posteriormente con las paradojas que producen: las intenciones - cognitivas y volitivas-; los actos, ilustrados por los movimientos corporales (o su ausencia) que dan forma a las intenciones; los resultados, que culminan, cierran y completan la acción; y las consecuencias, que se refieren al conjunto de acontecimientos que sólo emergen con la ejecución de la acción, sea simultánea o posteriormente a ésta ${ }^{23}$.

Este mapa heurístico será más adelante comparativamente utilizado en la obra de Elias, a fin de observar cómo el autor contempla, explícita o implícitamente, la naturaleza no intencional de lo social en su modelo teórico.

\section{NORBERT ELIAS: BREVE CONTEXTUALIZACIÓN DE SU OBRA}

Es frecuente que la vida y obra de grandes intelectuales queden injustamente eclipsadas u olvidadas por la comunidad académica. El extenso programa teórico de Norbert Elias constituye uno de los ejemplos más ampliamente reconocidos a lo largo del siglo xx: la vida de este «aléman-judío», cuya tenacidad se alimentaba de «la fe en que podía producir algo relativamente importante ${ }^{24}$, sólo obtuvo frutos tardíamente, a partir de los años ochenta, en un momento en que la rutina sociológica surgía impregnada de síntesis teóricas e integraciones paradigmáticas distintas. La confesión de Elias en uno de sus poemas: «o bien tendré un gran éxito, o bien pereceré» ${ }^{25}$, transparenta la premonición de la incierta proyec-

23 En la reconceptualización que hace del objeto de la sociología, donde pretende superar el individualismo metodológico y el estructuralismo de raíz durkheimiana, Lamo de Espinosa (1990) opera una distinción semejante entre acción —que detiene un significado subjetivo para el actor y termina donde él desea-y acto —que sigue produciendo consecuencias más allá del término de la acción y que comporta un significado real y objetivo no sólo para el actor, sino también para el observador-. A consecuencia de ello, el autor denomina resultantes al conjunto total de consecuencias objetivas derivadas de una acción, que pueden incluso entrelazarse o encadenarse dando origen al sustrato de la situación, que para el autor es seguramente diferente de las definiciones de las situaciones hechas por los actores sociales.

24 N. Elias, Mi trayectoria intelectual, Barcelona, Ediciones Península, 1995, p. 80.

25 N. Elias, op. cit., p. 23. 
ción académica que su obra lograría. Transcurridos once años de su muerte, la imagen de su creación intelectual es todavía tenue, aunque reconocible; a la vez que abre de forma paulatina vías teóricas alternativas.

Pese a la dificultad en rastrear los orígenes intelectuales del pensamiento de Elias, y en insertar su obra en las grandes tradiciones sociológicas clásicas, Bauman ${ }^{26}$ evalúa su labor sociológica de "conservadurista revolucionaria», por su intento paralelo de construir una teoría que anuncie el futuro de la ciencia social, pero sin renunciar a su vertiente decimonónica. Inscrito, como él mismo refiere, «por entero en la cadena generacional» ${ }^{27}$ de los intelectuales de su época — de los cuales se destacan Marx, Comte, Weber y Mannheim-, se esfuerza, sin embargo, en «desideologizar» las teorías sociológicas de todo el contenido de los partidismos políticos e ideales sociales vigentes ${ }^{28}$.

Esta neutralidad axiológica es formalizada a través de la construcción de una sociología histórica empíricamente fundamentada en El proceso de la civilización, que es considerada por algunos ${ }^{29}$ como su más ilustre empresa en describir las andaduras históricas en que se mueven y participan los individuos, y en su obra Compromiso y distanciamiento, donde elabora todo un corpus analítico en sociología del conocimiento que sirva de guía a la ética profesional. Todas sus demás obras no hacen más que especificar en mayor o menor amplitud temas inscritos en su opus magnum - El proceso de la civilización $n^{30}$ _. Pese a sus obras Sociología fundamental ${ }^{31}$ y Compromiso y distanciamiento ${ }^{32}$, que se centran más claramente en el problema ontológico de la redefinición del objeto de la sociología y en el problema epistemológico de cómo es posible adquirir

26 Z. Bauman, «The phenomenon of Norbert Elias», Sociology, 13, 1, 1979, pp. 117-125. Para un análisis comparativo de algunas de las convergencias y divergencias entre la perspectiva de Zygmunt Bauman en la modernidad/postmodernidad, y la de Norbert Elias en el proceso de civilización/decivilización, véase la interpretación de D. Smith, Norbert Elias \& Modern Social Theory, London, Sage Publications, 2001, pp. 114-133.

27 N. Elias, op. cit., p. 160.

28 Otro autor que explícitamente se destaca en este intento de «desideologización» es Georg Simmel. Para una lectura crítica sobre el modo cómo Elias recoge de la herencia simmeliana el concepto de «formas de socialización» y lo extiende a un nivel interpretativo histórico y cultural (civilización), véase C. Mongardini, «L'idée de société chez Georg Simmel et Norbert Elias», Cahiers Internationaux de Sociologie, 99, 1995, pp. 265-278.

29 Véase Z. Bauman, op. cit., 1979; R. Ramos Torre, «Del aprendiz de brujo a la escalada reflexiva: el problema de la historia en la sociología de Norbert Elias», REIS, 65, 1994, pp. 27-53; y J. M. González García, «Norbert Elias: literatura y sociología en el proceso de la civilización», REIS, 65, 1994, pp. 55-77.

30 N. Elias, El proceso de la civilización - Investigaciones sociogenéticas y psicogenéticas, Madrid, FCE, 2.ㄹ ed., 1993.

31 N. Elias, Sociología fundamental, Barcelona, Gedisa, 2.ㄹ ed., 1999.

32 N. Elias, Compromiso y distanciamiento, Barcelona, Península, 1990. 
tal conocimiento, sus demás trabajos incluyen, de hecho, extensiones temáticas de su obra magistral: la sociedad cortesana en la época de Luis XIV33, el problema del tiem$\mathrm{po}^{34}$, la civilización del deporte ${ }^{35}$, el envejecimiento y la muerte ${ }^{36}$, la guerra fría ${ }^{37}$ o el retrato de un genio ${ }^{38}$.

Delimitar el trasfondo temático eliasiano no es tarea fácil. Pese a las ambigüedades terminológicas, no siempre claras de interpretar, se pueden visualizar en sus obras dos ideas recurrentes: por un lado, la elaboración de una «sociología de los procesos», ilustrativa de un evolucionismo descriptivo de los largos procesos de la historia; y, por otro, su insistente tentativa en equilibrar el individualismo metodológico (el individuo sin sociedad) y el holismo (la sociedad sin individuos). Así, en la lógica analítica subyacente a los trabajos anteriormente reseñados se encuentra el intento eliasiano de esbozar una sociología procesual asentada en nociones dinámicas — procesos, figuración, interdependencia, poder- que intenten superar el estatismo conceptual de la sociología de su tiempo. Elias se enfrenta directamente contra el atomismo de la psicología y el estructural-funcionalismo de Talcott Parsons, por su énfasis en el análisis de los sistemas funcionales, $y$, como tal, desarrolla su propuesta alrededor de un modelo que contemple los sistemas interdependientes.

Mennell y Goudsblom ${ }^{39}$, dos de los teóricos más conocedores de la obra de Elias, identifican algunos principios transversales a su programa teórico:

1) El objeto de la sociología son los individuos en el plural, que se encuentran bajo varias formas de interdependencia, y cuyas vidas determinan y son a la vez determinadas por figuraciones sociales.

2) Estas figuraciones se hallan en flujo y movimiento continuos, siendo objeto de cambios tanto puntuales (sincrónicos) como más duraderos (diacrónicos).

33 N. Elias, La sociedad cortesana, Madrid, FCE, 2. a ed., 1993.

34 N. Elias, Sobre el tiempo, Madrid, FCE, 1989.

35 E. Dunning y N. Elias, Quest for excitement: sport and leisure in civilizing process, Oxford, Oxford University Press, 1986.

36 N. Elias, La soledad de los moribundos, México, FCE, 1987.

37 N. Elias, Humana conditio, Barcelona, Península, 1988.

38 N. Elias, Mozart. Sociología de un genio, Barcelona, Península, 1991. Esta interpretación temática es adaptada de R. Ramos Torre, op. cit., p. 28.

39 S. Mennell y J. Goudsblom (eds.), Norbert Elias. On Civilization, Power and Knowledge, Chicago and London, The University of Chicago Press, 1998. 
3) Los cambios a largo plazo inherentes a las figuraciones sociales ni son planeados ni previstos.

4) El desarrollo del conocimiento humano ocurre en las figuraciones sociales, siendo uno de los elementos principales de su desarrollo general.

En el siguiente apartado, trataremos de articular una reflexión que conjugue la propuesta anteriormente esbozada por Ramón Ramos acerca de las consecuencias no intencionales de la acción con el proyecto eliasiano ilustrado en los puntos 1 y 2 (cambios sincrónicos), para a continuación evaluar el modo en el que el autor desarrolla dentro de su sociología histórica las temáticas señaladas en los puntos 2 y 3 (cambios diacrónicos). Dejaremos de lado el análisis del punto 4, por no ser el objeto de este trabajo.

\section{HACIA EL ANÁLISIS DE LA SOCIOLOGÍA PROCESUAL ELIASIANA}

En la versión alemana, originalmente publicada en 1970, de la obra - Was ist Soziolo-

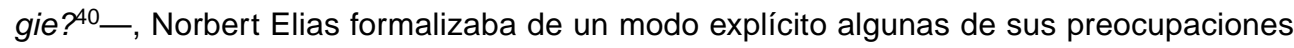
teóricas en el campo de la sociología. Su intento de superación del dualismo clásico entre «individuo» y «sociedad», representativos, respectivamente, del individualismo metodológico y del holismo, ha sido concretado en este libro a través de la metáfora de los «modelos de juego", ilustrativa de la dinámica de la realidad social. Es en esta obra donde algunos de los conceptos integrantes de su esquema teórico - figuración, interdependencia y poder- adquieren un rigor conceptual más consistente. En este sentido, se procurará emprender un primer análisis de su mapa conceptual, para posteriormente centralizar el modo como el autor circunscribe las «consecuencias no intencionales de la acción» como parte de lo que para él representa el objeto de la sociología, a saber, las figuraciones sociales.

Elias define -o semiesboza - el término «figuración» de un modo un tanto superficial en Sociología fundamental, aunque lo ejemplifique más detenidamente en La sociedad cortesana o en El proceso de la civilización. Este concepto trata de superar las polarizaciones clásicas entre «individuo/sociedad» y «acción/estructura» (ancladas en una imagen del homo clausus) y poner en el centro del análisis sociológico el entramado de las acciones humanas (individuales o colectivas) y sus interdependencias conflictivas o cooperativas (estructura). Como clarifica el propio autor, «el concepto de figuración posee además la excelencia (...) de no suscitar ni la idea de algo completamente cerrado o de

40 Editada en castellano bajo el título Sociología fundamental, op. cit. 
una armonía inmanente. El concepto de figuración es neutral. Puede referirse a relaciones de hombres armónicas, pacíficas y amistosas, así como inamistosas y conflictivas " ${ }^{41}$. Es más, las figuraciones son modelos de movimiento que se alimentan continuamente de las acciones individuales o colectivas, que, a su vez, son determinadas por equilibrios fluctuantes de poder que constituyen la peculiaridad estructural de cualquier relación social.

Como se observa aquí, el término figuración, que sugiere un dinamismo procesual inherente a la realidad social, procura igualmente sustituir la noción estructural-funcionalista de "estructura», y suplantar su carácter estático y de reificación social ${ }^{42}$. Con tal fin, Elias esboza una teoría en la que el modelo de las figuraciones sociales ocupa una posición central. Señala que éstas cambian a lo largo del tiempo de forma paulatina, surgiendo siempre de una figuración anterior, normalmente menos compleja y diferenciada. El cambio que ocurre en esa misma figuración - aunque sea dependiente de las acciones de los individuos ahí intervinientes - resulta en algo distinto de lo planeado o buscado intencionalmente. Así, las figuraciones se autonomizan y adquieren su lógica propia, reflejando el hecho de que de una acción individual resultan productos sociales no esperados, contingentes, pero que, asimismo, contienen en su esencia una estructura procesual ordenada que los singulariza.

La sociedad cortesana constituye el ejemplo histórico más inmediato del concepto de figuración. Recuperando datos empíricos de modo reconstrutivo, Elias esboza en el mapa de la sociedad francesa de la corte de Luis XIV el entramado de relaciones existentes entre el monarca y la alta aristocracia. La corte, en sí misma, ilustra una figuración concreta donde, a través de la etiqueta, el rey exhibe una pertenencia clara a la nobleza, al tiempo que se distancia de ésta para asegurar el poder. La posición del rey en la corte es única: es él quien tiene el poder de mediar en las luchas que se establecen entre los cortesanos, valiéndose de espías para saber de los deseos y ambiciones de cada uno. Esta vigilancia muda y sutil tenía como fin disolver la unión de posibles grupos de cortesanos que se formasen para atentar en contra del monopolio regio. Paralelamente, el monarca apoyaba a aquellos cuya posición en la corte era débil y que más dependían de sí para asegurar su presencia. Como vemos, la función de Luis XIV consistía en «una exacta ponderación de las relaciones de fuerza en su corte y un meticuloso balanceo del equilibrio de tensiones

\footnotetext{
41 N. Elias, La sociedad cortesana, Madrid, FCE, 1993, pp. 191-192.

42 En una comparación crítica sobre el concepto de «estructura» en varios paradigmas sociológicos —teoría de la figuración (Elias), estructural-funcionalismo (Parsons), marxismo (Marx) y teoría de la estructuración (Giddens)—, N. Mouzelis señala la necesidad heurística de utilizar integralmente este concepto tal como es definido por estos programas sociológicos, una vez que cada uno lo emplea en niveles analíticos distintos y no excluyentes entre sí. Cfr. N. Mouzelis, "On figuracional sociology», Theory, Culture \& Society, 10, 1993, pp. 239-253.
} 
que de esta manera se originaba en la corte como resultado de las presiones y contrapresiones» 43 .

Así pues, esta doble estrategia del rey - apoyar a los débiles y vigilar a aquellos que potencialmente representaban un peligro para su posición- le permitía equilibrar fuerzas y legitimar su dominio en la cadena de interdependencias de la corte francesa, incluso no poseyendo características carismáticas inherentes a cualquier líder. Con esto, Luis XIV mantuvo bajo su control las consecuencias que podrían advenir de una probable unión de fuerzas entre los cortesanos, alimentando continuamente los conflictos internos entre ellos puesto que ésta era su mejor arma para permanecer con el monopolio del poder. Pero Luis XIV, para asegurar su dominio, tenía igualmente que someterse a la figuración cortesana que lo rodeaba: su vida privada era absorbida por la etiqueta y mirada de la corte, a quienes debía obligatoriamente conferir oportunidades de prestigio y poder personal. La conservación y solidificación de su dominio se produjo paulatinamente a lo largo de su reinado, de tal forma que su hijo Luis XV no experimentó ninguna amenaza externa sobre su posición. El doble juego en el que se encontraba inscrito el rey, y el cumplimiento de sus normas sociales, le permitieron reinar y desarrollar en Francia una innegable centralización estatal.

Tras esta ejemplificación histórica del concepto de figuración, analicemos enseguida cómo Elias $^{44}$ establece una analogía entre las figuraciones sociales y seis modelos de juego, que, al igual que aquéllas, son orientados por equilibrios diferenciales de poder, y que sostienen una lógica «regulada» y «ordenada», con una autonomía propia y distinta de aquella característica de la psicología o biología ${ }^{45}$. El poder es para el autor un concepto central, con una naturaleza polimórfica, constitutivo de la singularidad estructural de todas las relaciones humanas, y que se encuentra, cómo mínimo, presente en las relaciones bipolares y en la mayoría de las relaciones multipolares. Detengámonos ahora aquí un momento, para evaluar cómo Elias conceptualiza esta noción.

El concepto de poder, para Elias, se relaciona, por un lado, con las figuraciones no intencionales existentes entre las intenciones y, por otro, con la cuestión de cómo interdependencias no intencionales pueden ser usadas intencionalmente por los seres humanos en el curso de los procesos sociales. Es decir, el término poder traduce un intento de superación

\footnotetext{
43 N. Elias, La sociedad cortesana, op. cit., p. 163.

44 N. Elias, Sociología fundamental, op. cit.

45 La distinción epistemológica entre ciencias naturales y ciencias sociales ha sido ampliamente desarrollada en su obra Compromiso y distanciamiento.
} 
epistemológica, constituyendo un punto de intersección entre estructura y acción. El grado por el cual los individuos pueden moldear los procesos sociales conforme a sus deseos y el grado en que estos mismos procesos -incluyendo las consecuencias a ellos asociadas- se desvían de sus intenciones dependen, para el autor, del diferencial de poder.

Con esto, en el cuadro teórico eliasiano, dicho concepto se refiere a una capacidad no del actor, pero sí a una propiedad estructural de una relación social, i.e., ilustra una relación entre relaciones. El poder es así entendido como el cociente de dependencias recíprocas existentes en cualquier relación humana. En este sentido, el término monopolio posee una importancia central. Una situación social representada por un monopolio se refiere a una situación donde la dependencia de una de las partes por la otra alcanza un máximo, mientras la dependencia inversa es minimizada.

Junto con la noción de poder, Elias crítica el significado asumido en la teoría estructuralfuncionalista de cariz parsoniana del concepto de «función». El autor se posiciona en contra de su carácter unilineal ( $A$ condiciona $B »)$, refiriendo que las funciones sociales poseen una naturaleza dialéctica y bidireccional traducible en una serie de interdependencias coactivas. Así, poder y función son conceptualizados en esta obra como nociones íntimamente relacionadas entre sí y que implican una relación procesual de dependencia en la que tanto $A$ influye a $B$ como $B$ a $A$, en la medida en que $A$ tiene poder sobre $B$ a la vez que lo necesita, y viceversa. Con esto, Elias solidifica su énfasis en los sistemas de interdependencia, enormemente críticos con el tono parsoniano que poseen los sistemas funcionales a la hora de explicar la realidad social.

La distinción analítica entre sistemas funcionales y sistemas de interdependencia merece aquí una clarificación puntual. En la obra ya anteriormente citada de Raymond Boudon ${ }^{46}$, afirma que las acciones individuales pueden incluirse tanto en sistemas funcionales - cuando la acción es desarrollada en el contexto de un papel específico-como en sistemas de interdependencia — donde las acciones entre individuos carecen de un papel determinado para realizarse- En convergencia con la perspectiva de Elias —con la cual Boudon se identifica-, los sistemas de interdependencia producen «efectos emergentes», es decir, consecuencias no incluidas en las intenciones individuales, y que adoptan diversas formas sociales que se escapan al control.

Analicemos ahora más detenidamente los varios tipos de modelos de juego diseñados metafóricamente por Elias, cuyas «reglas son artificiales y se corresponden, de todos modos, en sus formas más simples a juegos como el ajedrez, el tresillo, el fútbol, el tenis o cuales-

46 R. Boudon, op. cit. 
quiera otros juegos "reales" 47 , y procuran subrayar el carácter procesual y no planeado inherente a las relaciones entre individuos interdependientes, cambiante según la distribución de poder ${ }^{48}$.

Veamos el primer caso - 1.a)—. A (jugador fuerte) tiene un alto grado de control sobre B (jugador flojo). Así, aunque B tenga que orientar sus jugadas (acciones) de acuerdo con lo que $A$ juega, este último también tiene que tener en cuenta las jugadas de su compañero. Es más, por un lado, A tiene poder sobre B, pero, por otro, también tiene poder sobre el proceso del juego y de su resultado. Lo que resalta en este modelo — que se procura situar más allá de las teorías de la acción- es que aunque éste se halle en gran medida estructurado por las intenciones y las acciones de un individuo, dicho individuo tiene que tener en cuenta el entramado de interdependencias en que se inscriben, dado que el otro actor también participa activamente en el proceso del juego. Veamos el esquema que sigue ${ }^{49}$.

Modelo 1.a)

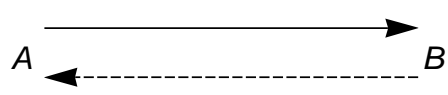

$\longrightarrow$ Centralidad e intensidad del poder poseído

- Capacidad de participar en la partida e intensidad disminuida de poder

Articulando este modelo con el mapa operativo anteriormente expuesto por Ramos ${ }^{50}$, observamos que, en esta situación de interdependencia simple, la probabilidad de que ocurran consecuencias no previstas y no deseadas — casos 1.d), 2.d) y 3.d) - es diminuta en tanto en cuanto el poder de la partida y las acciones de $B$ estén centralizadas en el jugador A. De hecho, el diferencial de poder - central para Elias y no contemplado por Ramos- es el hilo organizativo de las interdependencias sociales y de las consecuencias intencionales/no intencionales que de ahí advienen. Con todo, y como veremos en los ejemplos siguientes, Elias atribuye los cambios ocurridos en el diferencial de poder a la propia natura-

47 N. Elias, op. cit., p. 86.

48 En El proceso de la civilización aparece otro tipo de aclaración del término «figuración» ilustrado en la metáfora de la danza (cfr. p. 45).

49 Este esquema y los que siguen ilustran meramente el diferencial de poder poseído en una relación de interdependencia. El entramado de consecuencias que resultan de estas interrelaciones será presentado en un cuadro final en articulación con el modelo de Ramón Ramos.

50 Cfr. R. Ramos Torre, “Una aproximación...», op. cit. 
leza imprevista de lo social, que, con su curso no intencionado ni planeado, hace emerger gradualmente modelos complejos y ordenados que constriñen a los individuos para actuar en una determinada dirección que termina por escaparse de sus propios designios.

Analicemos ahora otro caso -1.b) - Supongamos que el diferencial de poder entre A y B disminuye —es decir, A pierde la fuerza y B aumenta la suya -; aquí, la capacidad de cada uno para determinar la conducta del otro se reduce y, así, disminuirá el poder de las intenciones o planes que se hayan delimitado en el proceso del juego. Como el propio autor indica, "tanto más adquiere el juego un carácter de proceso social y pierde el de ejecución de un plan individual; en tanto mayor medida resulta, con otras palabras, del entramado de jugadas de dos individuos un proceso de juego que no haya sido planeado por ninguno de los dos jugadores ${ }^{51}$. Este modelo se puede visualizar en el esquema que sigue.

Modelo 1.b)

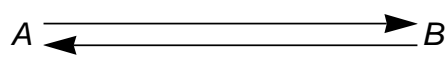

$\longrightarrow$ Intensidad equitativa en el poder poseído

O sea, aquí el proceso mismo del juego y las consecuencias que tienen las jugadas de cada uno de los jugadores se autonomizan y asumen un curso singular en tanto en cuanto el diferencial de poder entre los dos individuos se reduzca. Nótese que, en articulación con el esquema de Ramos, el grado de probabilidad para que ciertas consecuencias no previstas ni deseadas ocurran — casos 1.d), 2.d) y 3.d) - aumenta en proporción a la disminución del diferencial de poder entre los jugadores.

Observemos el siguiente caso -2.a)-. Aquí, nos encontramos con un modelo de grupo bipolar en que A juega simultáneamente con B, C y D, siendo claramente superior a cada uno de ellos y controlando así el curso general del juego. Nos topamos aquí con la presencia de una serie de juegos entre dos individuos ( $A$ y $B, A$ y $C, A$ y $D$ ) con un equilibrio de poder desigual. Este ejemplo se puede, con todo, ampliar a un modelo de grupo multipolar -2.b)—: A juega simultáneamente la misma estrategia con $B, C$ y D, y tiene más poder que éstos. Pero si B, C y D se unen pacíficamente entre sí contra A, consiguen disminuir su fuerza y desestabilizar su control sobre el curso del juego. También puede suceder -modelo 2.c)— que este último modelo se consolide a lo largo del tiempo y, así, que el diferen-

51 N. Elias, op. cit., p. 96. 
cial de poder se anule (es decir, que haya una fuerza equilibrada de poder entre $\mathrm{A}$ y $\mathrm{B}, \mathrm{A}$ y $C, A$ y $D$ ). Los siguientes esquemas permiten clarificar estos tipos de interdependencias.

Modelo 2.a)

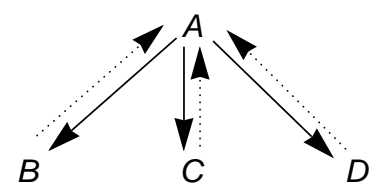

$\longrightarrow$ Centralidad e intensidad del poder poseído

- Capacidad de participar en la partida e intensidad disminuida de poder

Modelo 2.b)

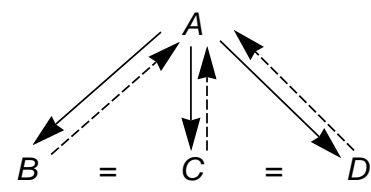

$=\quad$ Relación de unión entre los individuos

$\longrightarrow$ Disminución de la intensidad de poder poseído

--- Aumento de la intensidad de poder poseído

Modelo 2.c)

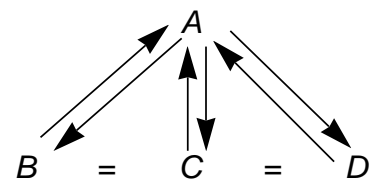

$=\quad$ Relación de unión entre los individuos

$\longrightarrow$ Intensidad equitativa del poder poseído 
Analicemos un último caso donde estas relaciones se complejifican —modelo 2.d)—: dos grupos con fuerzas de poder iguales juegan entre sí - B, C, D y E contra U, V, W y XAquí, como ninguna de las acciones puede ser considerada o explicada individualmente, el curso o proceso del juego resulta siempre del entramado anterior y del entramado futuro que se espera que ocurra en las acciones de ambas partes. Se observa mejor esta dinámica a través del esquema que sigue.

Modelo 2.d)

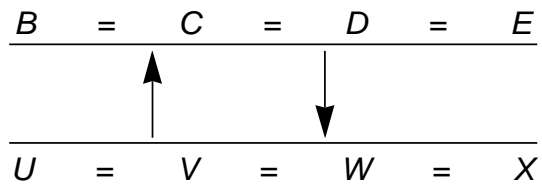

$=\quad$ Relación de unión entre los individuos

$\longrightarrow$ Fuerza equitativa de poder poseído

Confrontando ahora cada uno de estos modelos con la propuesta de Ramos, observamos que el modelo 2.a) posee una lógica semejante a 1.a): el poder está concentrado en un solo jugador (A), que tiene la capacidad de reducir las consecuencias no previstas ni deseadas - casos 1.d), 2.d) y 3.d) de Ramos- a lo largo del juego. Pero si el diferencial de poder progresivamente se equipara o anula - modelos 2.b) y 2.c)—, la probabilidad de ocurrencia de los casos 1.d), 2.d) y 3.d) señalados por Ramos aumenta sustancialmente; llegando el diferencial de poder a constituirse como la regla procesual del entramado de interrelaciones sociales en el modelo 2.d). Nótese aun que la probabilidad de que existan casos de sobreintencionalidad y contraintencionalidad es bastante fuerte, dado que nos encontramos en presencia de un modelo que presupone una dialéctica relacional importante, en el que es la propia naturaleza interdependiente de la estructura social lo que directamente condiciona las jugadas ya no individuales, pero sí sociales.

Si los modelos anteriormente analizados de 2.a) a 2.d) se desarrollaban en un plano, observemos ahora lo que ocurre en otro modelo de juego entre varias personas en diversos planos. Aquí, el número de jugadores participantes aumenta constantemente, de tal forma que si un jugador individual quisiera introducirse en el juego tendría que esperar bastante, siéndole cada vez más difícil hacerse una imagen clara de la cambiante figuración de la situación. Observamos que la impenetrabilidad del juego se torna progresivamente consciente para cada uno de los participantes, así como la manera cómo aquél se desarrolla 
cambiando en una dirección específica. La complejidad y la opacidad de las relaciones sociales en este proceso provocan que el juego se vaya desorganizando, siendo necesaria su reorganización. Aquí puede optarse, según Elias, entre algunas posibilidades:

1) La desintegración del grupo origina otros más pequeños que hacen que: a) los grupos fraccionados se alejen más unos de los otros, y que los jugadores sigan jugando independientemente unos de los otros; b) estos grupos puedan formar una nueva configuración a partir de grupos más pequeños e interdependientes.

2) El grupo pueda mantenerse integrado, pero forme una configuración más compleja; así, de un nivel de actuación se puede pasar a dos niveles distintos.

De esta última situación, Elias esboza otros dos tipos de modelos en los que los jugadores no juegan directamente unos con los otros. El primer tipo -3.a) - lo define como oligárquico, y es constituido por dos pisos (o dos niveles) que podrían ramificarse en otros más, notoriamente distintos por los diferenciales de poder, que son mucho más favorables al Piso 2 (de arriba) que al Piso 1 (de abajo). Observemos el siguiente esquema.

Modelo 3.a)

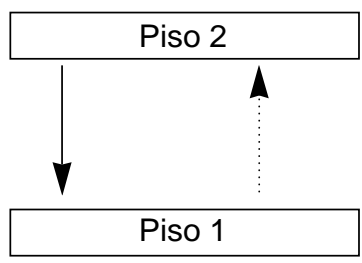

$\longrightarrow$ Centralidad e intensidad del poder poseído

- Capacidad de participar en la partida e intensidad disminuida de poder

Así, sólo los individuos del Piso 2 tienen una participación directa y activa en el curso del juego (monopolio de acceso), poseen una imagen de la figuración del juego más amplia y completa y éste se les presenta transparente, de tal forma que sus acciones son orientadas por estrategias precisas. Pero Elias advierte que la imagen de transparencia poseída por los individuos es ilusoria e irreal. La transparencia sólo se adquiere con una investigación social más detenida, que apenas es posible cuando "se llega a un nivel de desarrollo de la sociedad en el que los hombres pueden ser conscientes al mismo tiempo de su igno- 
rancia, es decir, de la relativa opacidad del juego en el que practican sus jugadas, y la posibilidad de reducir esa ignorancia mediante la investigación sistemática»52.

Aunque Elias introduzca en este modelo un componente institucionalizado de poder, su lógica ejecutiva es convergente con aquella observada en los modelos 1.a) y 2.a); es decir, la probabilidad de que no ocurran las consecuencias ilustradas por Ramos en 1.d), 2.d) y 3.d) es elevada, al estar el monopolio del curso del juego centralizado en uno de los niveles.

Otro tipo de juego es el democrático - 3.b)—. Aquí, la fuerza de los jugadores del piso de abajo aumenta progresivamente enfrentándose a la fuerza de los jugadores de arriba, de forma que el diferencial de poder tenderá a mayores oscilaciones en uno u otro sentido. Así, las coacciones a que están sujetos los individuos del piso de abajo por parte de los de arriba son cada vez menos visibles. Esto también se refleja en el modo en como la representación táctica del juego se modifica, y se construyen estrategias integradas más globales para hacer frente a la autonomía relativa con que discurre el partido. El esquema siguiente es ilustrativo de esta situación.

Modelo 3.b)

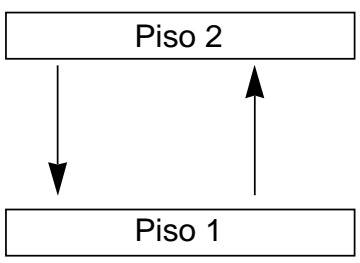

$\longrightarrow$ Intensidad equitativa del poder poseído

Relacionando este modelo con el de Ramos, resalta una vez más el hecho de que cuanto más se equilibre el diferencial de poder, más probable es la ocurrencia imprevista e indeseada de las consecuencias destacadas en 1.d), 2.d) y 3.d), dado que el monopolio del juego se reparte entre los individuos ${ }^{53}$.

52 N. Elias, op. cit., p. 102.

53 Retomando el resumen analítico presentado en la nota 24 (cfr. p. 8) de la propuesta de E. Lamo de Espinosa sobre la reconceptualización de la acción social como objeto de la sociología, es fundamental extender aquí su concepción sobre los procesos reflexivos que se producen y pueden conducir a consecuencias no intencionales de la acción intencional en contex- 
Tras esta exposición, puede observarse en los modelos constituidos por Elias que de acciones intencionales discurren consecuencias que no han sido planeadas y que escapan a cada uno de los agentes, los cuales se dan progresivamente cuenta de su incapacidad para controlarlas. En los modelos anteriormente analizados se ha transitado de un proceso de juego que depende de jugadas individuales a su autonomización, que motiva un curso no planeado, al cual cada uno de los jugadores se tendrá que adaptar. Mejor dicho, para Elias, las consecuencias no intencionales son parte de la propia figuración social - condiciones no intencionales, aunque estructuradas - en que se mueven los individuos, determinando las intenciones y acciones de cada uno de ellos.

La articulación a lo largo del texto de estos modelos metafóricos con el mapa operatorio de Ramos evidencia la gradación creciente de complejidad social, que partiendo de las intenciones individuales iniciales evoluciona hacia un entramado de interdependencias que naturalmente se agregan a las intenciones iniciales - sobreintencionalidad - o las desplazan — contraintencionalidad- Las intenciones y acciones en la perspectiva eliasiana son, pues, dependientes del curso de la propia figuración en que se inscriben.

Aunque Elias no distinga elementos cognitivos y elementos volitivos, ni Ramos incorpore en su análisis el concepto de poder, es posible integrar resumidamente la esquematización hecha por cada uno de los autores en el siguiente esquema. La dirección de las flechas indica la probabilidad de ocurrencia en cada modelo de cierto tipo de consecuencias intencionales/no intencionales, dependiendo siempre del diferencial de poder poseído por los agentes del sistema de interdependencia.

tos de acción monopolísticos y democráticos. Recordando que para el autor las resultantes de las acciones son un producto final del entrelazamiento de varias acciones, y que estas mismas resultantes cambian según el tipo de recursos (económicos, poder político, poder simbólico, etc.) que cada actor posee, Lamo de Espinosa define dos tipos de extremos de un continuo social -el contexto democrático, en que el peso de cada acción es equivalente a las demás-y el contexto monopolístico, en que los recursos están concentrados en las manos de sólo uno o de pocos actores. En el primer caso, obsérvese que la resultante global de las acciones es menos previsible para cada actor, mientras en el segundo la resultante final posee un grado esperado de certeza. Comparada esta propuesta con la de N. Elias, se observa una analogía entre el contexto monopolístico definido por Lamo de Espinosa con el modelo 3.a) de Elias, y entre el contexto democrático y el modelo 3.b) anteriormente presentado. Véase E. Lamo de Espinosa, «La sociedad reflexiva. Ciencia social y sociedad del conocimiento», en E. Lamo de Espinosa, J. M. González García y C. Torres Alberto (eds.), La sociología del conocimiento y de la ciencia, Madrid, Alianza Editorial, 1994, cap. 24, pp. 620-625. 
Esquema síntesis: Articulación del modelo de R. Ramos y N. Elias

$A$

1.a) Prevista y querida

2.a) Se agrega a lo previsto y a lo querido

3.a) Previsible y deseable
$B$

1.b) No prevista y querida

2.b) Desplaza a lo previsto y se agrega a lo querido

3.b) Imprevisible y deseable
C

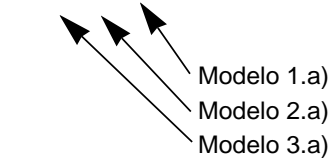

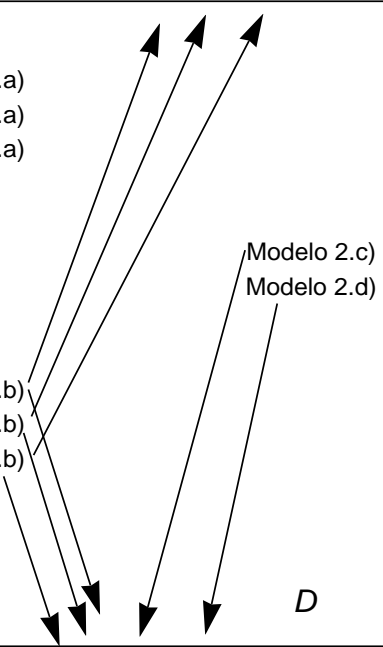

1.d) No prevista ni querida

2.d) Desplaza a lo previsto y a lo querido

3.d) Imprevisible e indeseable

Leyenda:

- Las letras mayúsculas que encabezan los cuadros se refieren a la agregación de todas las letras minúsculas incluidas en los cuadros 1, 2 y 3 de Ramos (véanse pp. 6-7).

- Modelos 1.a) - 3.a): el poder está centralizado en un solo individuo.

- Modelos 1.b) - 3.b): el poder está menos centralizado en un solo individuo.

- Modelos 2.c) - 2.d): el poder está equitativamente repartido entre los individuos.

Analicemos ahora más atentamente las limitaciones teóricas que posee el concepto de figuración. En primer lugar, y como hemos visto, en todo este proceso interviene directamente el diferencial de poder como elemento central y organizativo de todas las relaciones humanas. Pero cuando Elias indaga "¿qué es realmente lo que interrelaciona a los hombres en figuraciones?, ${ }^{44}$, la respuesta dada es un tanto superficial e insatisfactoria: según el autor, lo que vincula a los hombres en las figuraciones sociales son motivos impersonales (i.e., económicos y que implican una dependencia funcional), estatales (i.e., militares y que suponen la protección frente a la amenaza externa) y personales (sexuales y afectivos,

54 N. Elias, op. cit., p.160. 
expresados por distintas formas simbólicas). Es decir, lo que reuniría para Elias a los seres humanos alrededor de varios tipos de figuraciones serían razones diversas - económicas, militares o afectivas-, que condicionarían en mayor o menor grado las acciones individuales según el diferencial de poder poseído. Con todo, el autor en la obra Sociología fundamental apenas describe la manera en que cada uno de estos elementos puede contribuir a englobar los individuos en figuraciones concretas, pero no explica de qué forma estos elementos los impelen y constriñen a la hora de actuar. De hecho, el modo como los elementos cognitivos y emocionales que anteceden la ejecución de una acción concreta se entremezclan con el concepto de poder queda escasamente formalizado en los modelos de juego eliasianos, dejando aquel término definido de forma abstracta y excesivamente ecléctica en el modelo de interdependencias que procura construir ${ }^{55}$.

En segundo lugar, y como ha sido anteriormente mencionado, la teoría de las figuraciones constituye un intento de superar la escisión clásica individuo/sociedad, siendo extensible desde relaciones sociales micro (p.e., las interdependencias en un grupo terapéutico entre médico y pacientes) a otras macro y globales (p.e., las relaciones económicas entre Estados). Se observa aquí claramente el deseo eliasiano de dotar a este término de un eclecticismo operatorio que posibilite la descripción de relaciones sociales de diverso tipo (micromicro, micro-macro, macro-macro). Con todo, y tal como lo teoriza Elias, no parece que el concepto de figuración logre resolver el dualismo que se proponía salvar, siguiendo anclado en un nivel macro y meso. Es justamente en este contexto donde conviene indagar si ciertos elementos descriptivos del mundo de la vida (intersubjetividad, estados de conciencia, reflexividad, motivos, deseos y emociones) analizados por sociologías interaccionistas y fenomenológicas, y largamente desarrollados en las obras de G. H. Mead, H. Blumer y A. Schutz, pueden ser explicados bajo la potencialidad analítica de este concepto. Al desarrollar una analogía entre las figuraciones y los modelos de juego, Elias señala que éstas no pueden existir independientemente de las acciones de los individuos, y viceversa, y que como tales no reifican la realidad social. Pero lo que se defiende aquí, en coherencia con la teoría interaccionista, es que estas figuraciones del juego sí pueden existir como ideas, como mapas mentales, como símbolos, independientemente de su realización ejecutiva. En este sentido, y contra Elias, estos constructos mentales son reales y existen objetivamente en el exterior de los individuos, integrando el aparato simbólico-cultural de una sociedad dada ${ }^{56}$. Nuestra tesis aquí es que el concepto de figuración no es extensible al aná-

\footnotetext{
55 Elias da una respuesta mucho más satisfactoria a esta cuestión en El proceso de la civilización, donde analiza diacrónicamente el modo como las heterocoacciones pasan a autocoacciones a lo largo de la historia occidental. De hecho, Elias cumple mejor sus objetivos empíricos que los teóricos. Con respecto a estos últimos, no logra definir con precisión ni profundizar debidamente los conceptos que propone.
}

56 Para una interpretación convergente, véase D. Layder, «Social reality as figuration: a critique of Elias' conception of sociological analysis», Sociology, 20, 3, 1986, pp. 367-386. 
lisis de estos elementos, no logrando integrar dialécticamente la naturaleza perceptiva y representacional de las intenciones que anteceden una determinada acción con la estructura social en que adquieren visibilidad, y que es condicionada y les condiciona a la vez.

En tercer lugar, y en relación con el punto anterior, Elias no mira o descuida las consecuencias intencionales de la acción intencional (contrariamente a los interaccionistas simbólicos, para quienes el hecho central son las intenciones de los sujetos), y su preocupación por los individuos parece reducirse al análisis de las posiciones cambiantes de poder poseídas por ellos en el proceso de figuración. En su intento «antifuncionalista» de superar el estatismo y el carácter causal de la realidad social - y que para ello subraya la autonomía de las consecuencias no intencionales en la realidad social frente a las intenciones de los actores-, el autor se olvida de definir con más rigor la definición subjetiva de la situación en que se mueven los individuos. Elias piensa escapar a este problema sosteniendo que «las figuraciones son tan concretas como los actores», es decir, que unas y otros se interrelacionan de forma dialéctica y continua. En este sentido, retira libertad de acción al actor, apaga sus deseos y motivos, y los reduce a condicionamientos de la figuración social e histórica en la que se mueve. Esta idea está claramente ilustrada en el hecho de que, para Elias, el carácter racional de la acción del actor es un producto de las cadenas de interdependencia en que se mueve: cuanto más se encuentra éste encerrado en éstas, más racionales y menos autónomos se vuelven sus comportamientos ${ }^{57}$.

El tema de las consecuencias no deseadas de la acción en la tesis eliasiana merece aquí una aclaración final. Para Elias, como ha sido dicho, las cadenas de interdependencias del proceso de figuración constituyen y son a la vez constituidas por consecuencias no intencionales. El actor, mientras esté inscrito en un entramado de interdependencias, nunca posee una idea global o transparente de la situación; de hecho, la opacidad en que se mueve y actúa le imposibilita ver claramente la totalidad de la situación para definir una representación social objetiva y completa que preceda a su propia actuación —véase, por ejemplo, el caso del modelo 2.d)—. Más aún, la complejidad y opacidad subyacentes al entramado de interdependencias aumentan si el numero de personas crece (argumento cuantitativo) o si en la figuración del proceso hay diferenciales de poder iguales o desiguales (argumento cualitativo).

Helena Béjar ${ }^{58}$, en el tratamiento de las consecuencias no intencionales, parte de la distinción entre: a) consecuencias no deseadas de la acción, que conducen a las paradojas de la acción social; b) y el extrañamiento social, que resalta el carácter opaco de la sociedad

57 Esta idea está explícitamente demostrada a un nivel diacrónico en El proceso de la civilización.

58 H. Béjar, La cultura del yo - Pasiones colectivas y afectos propios en la teoría social, Madrid, Alianza Editorial, 1993. 
bajo una visión holista. Béjar interpreta la tesis de Elias como inscrita en esta última definición, señalando que la preocupación del autor en el tema del cambio social es justamente el hecho de que la sociedad sea "convertida en una facticidad cuyo origen escapa a los hombres ${ }^{59}$. En nuestra opinión, en ambas posturas diferenciadas por Béjar resalta la idea de que el entramado de las acciones individuales produce consecuencias no deseadas («el todo es distinto de la suma de las partes»), y esto genera bien características paradójicas, bien opacidad social. En este sentido, y apoyándonos en nuestra interpretación de Elias, para el autor el carácter opaco de la sociedad es a la vez un a priori y un producto final; es decir, los actores sociales actúan y orientan sus prácticas inscritos en una figuración social ya por sí misma opaca, no visible, lo que conduce a que casi siempre adopten una u otra decisión comportamental, siempre carente de toda la información necesaria para actuar. Es imposible, como dice el propio Elias, pensar que se posee toda la información al tomar una decisión, dado que es esa misma ignorancia de la situación lo que imposibilita una definición correcta del presente y una previsión lúcida del futuro ${ }^{60}$.

La tarea del sociólogo sería justamente, para el autor, la de «zapador de mitos», en tanto en cuanto tendría que hacer visible esa opacidad, de sacar a la luz la figuración de los procesos y su desarrollo dinámico en la sociedad, con el propósito de controlar progresivamente el devenir histórico y social. Merece aquí la pena reproducir textualmente sus palabras cuando dice que «la tarea de la investigación sociológica consiste en aplicar el entendimiento humano a estos procesos ciegos y carentes de dirección; la tarea consiste en explicarlos y a través de ello, posibilitar a los hombres la orientación en los entramados producidos por sus propias acciones y necesidades, que parecen impenetrables, y en posibilitar asimismo un mejor control de tales procesos "61.

Concluimos este apartado tras cumplir el objetivo propuesto en los puntos 1 y $2^{62}$ : enseñar el modo cómo Elias conceptualiza el objeto de la sociología — los individuos en interdependencia-, que se inscriben y actúan sincrónicamente en determinadas figuraciones sociales, cambiantes en complejidad y estructura interna, y de las cuales resultan productos finales no incluidos en sus intenciones y que se escapan a su control.

59 H. Béjar, op. cit., p.128.

60 Merton (1980: 177-185) sostiene una opinión convergente en este tema, aunque amplíe el análisis de las causas de las $\mathrm{CNI}$ a otras fuentes más allá de la propia ignorancia del actor -error, imperiosa inmediatez de interés, valores básicos y predicciones autodestructivas.

61 N. Elias, op. cit., p.186.

62 Cfr. p. 9. 


\section{LA CIVILIZACIÓN COMO PROCESO: MATICES TEÓRICOS DE SU EVOLUCIÓN NO INTENCIONAL}

En el apartado anterior procuramos ilustrar cómo el concepto de figuración preside la obra de Elias, constituyendo la promesa de liberarse sociológicamente tanto de un individualismo sociológico como de un estatismo holista ilustrativo de un orden social reificado. En este sentido, los modelos de juego anteriormente analizados representan una metáfora descriptiva del modo en el cual coexisten en la estructura procesual eliasiana las acciones intencionales y aquellas más carentes de orden, contingentes - no intencionales- y que imposibilitan la determinación de acontecimientos futuros bajo el control de los individuos. Así, para Elias, los procesos sociales e históricos resultan del anonimato de las acciones individuales, o, mejor dicho, ilustran los efectos no previstos de la interdependencia social: "pero es evidente que en ningún momento ha habido seres humanos individuales que hayan tratado de realizar esta transformación, esta "civilización", de modo consciente y "racional" por medio de una serie de medidas que persigan tal objetivo. (...) Es impensable que el proceso civilizatorio haya sido iniciado por seres humanos capaces de planificar a largo plazo y de dominar ordenadamente todos los efectos a corto plazo, ya que estas capacidades, precisamente, presuponen un largo proceso civilizatorio»63.

Retomaremos ahora aquí el objetivo señalado inicialmente en los puntos 2 y $3^{64}$ : analizar el modo cómo el autor desarrolla una concepción del cambio diacrónico de las figuraciones sociales como un proceso que no es un producto intencional de la voluntad humana ni el resultado de una planificación racionalizada, aunque siga un orden y lógica particular.

La ejemplificación diacrónica de la teoría figuracional se encuentra materializada en El proceso de la civilización. Apoyado documentalmente en libros históricos de modales, Elias enseña detenidamente en esta obra cómo un tipo particular de figuración —la sociedad medieva — evoluciona hacia otro tipo más civilizado — la sociedad cortesana—y éste, a su vez, hacia otro - la sociedad burguesa - a través de un proceso social paulatino de represión de los instintos naturales y de los impulsos, que culmina con la solidificación de una civilización normalizada, controlada y socializada, que exhibe una amnesia colectiva de su propia contención instintiva materializada a lo largo de la historia occidental.

El proceso de la civilización marca el tránsito desde el heterocontrol hasta el autocontrol; esto es, el paso desde la coacción externa como fundamento de la conducta individual hasta la autocoacción como fundamento de la misma. Este proceso, que Elias describe

63 N. Elias, El proceso de la civilización..., op. cit., p. 449.

64 Cfr. p. 9. 
para Europa Occidental, arranca analíticamente en el periodo bajomedieval para prolongarse, en su estudio, hasta bien entrado el siglo XIX. La civilización del comportamiento corre en paralelo a otros procesos que la hacen posible: la cortesanización de los guerreros, la mudanza desde una economía natural a una dineraria, la concentración del poder político en detrimento de los poderes locales fragmentarios, la traslación del uso de la violencia en manos de los particulares a manos del Estado, la propia construcción del Estado y la progresivamente creciente diferenciación social.

Inicialmente, Europa está constituida por una infinidad de pequeñas unidades políticas que compiten entre sí con la intención de imponer su hegemonía en porciones de territorio que se quieren cada vez mayores. Tal competencia hace que, poco a poco, unas pocas unidades políticas vayan incrementando su tamaño y área de influencia, mientras que las restantes o bien son absorbidas o bien desaparecen. Encontramos aquí el germen de los futuros Estados. El Viejo Continente comienza a definirse territorialmente con base a la simple confrontación bélica, confrontación para la que resulta imprescindible el guerrero como tipo humano característico de un tiempo esencialmente violento.

Estamos ante hombres espontáneos afectivamente, extremos sentimentalmente, que encuentran en la violencia que puedan ejercer aquellos cuantos les rodean el freno y límite para su propia conducta. Mas el Estado comienza a delinearse y, con él, una progresiva monopolización de la fuerza militar que le hará mostrarse cada vez menos dependiente del concurso de los señores feudales en las guerras. Al frente de ese Estado que se va esbozando se perfila un monarca absoluto que acogerá en el seno de una corte naciente a todos aquellos nobles que han perdido su utilidad militar y deben vincularse entre sí, no ya a través de la guerra, sino mediante el prestigio. Éstos han de ajustar su conducta a la de un número mayor de personas, ocurriendo en este ajuste que uno debe volverse dueño de su propio comportamiento. Así, cuando la competencia entre los hombres no puede tener como eje a la violencia, aquélla comenzará a articularse en torno al prestigio y la distinción. La pretensión de distinguirse fomenta el desarrollo de modelos de sociabilidad en los comportamientos cotidianos, cada vez más refinados, dulcificados y depurados - modo de comer en la mesa, de dormir, de afeitarse, de defecar-, patrimonio inicial de los estratos más elevados aunque desplazándose en sentido descendente por la escala social, al tiempo que continúan elaborándose más modelos con el afán de distinguirse. A partir de este instante será el miedo a la degradación pública lo que empuje a los individuos a controlar su comportamiento, interiorizando todas y cada una de las coacciones que una vez fueron externas hasta convertirlas en una segunda naturaleza, hasta convertirlas en coacciones que se activen de manera automática. La normalización de este proceso favorece la aparición de un tipo de personalidad individualista, más empático, y capaz de autocontrolar los impulsos y emociones en varias situaciones sociales e individuales. 
Esta interpretación eliasiana de la civilización encuentra su raíz en Freud, para quien la civilización se construye bajo la renuncia de los instintos primarios y de su autodisciplina; a la vez que se aleja de la postura weberiana, más conceptuada entre la clase sociológica de aquel tiempo, y que señala la centralidad de la racionalidad como motor transversal del cambio histórico-social.

Pero ¿cómo se puede explicar el desarrollo de la estructura de estos procesos sociales a lo largo de la historia si éstos no son el producto intencional de las acciones individuales? Como hemos dejado anteriormente explícito, Elias contesta a esta cuestión afirmando que este desarrollo encuentra su fuerza en la dinámica de los procesos de competencia y de monopolización, transversales a las distintas formas de figuración social. Así, en situaciones de competencia en las cuales la existencia social del individuo o la unidad social a la que éste representa están amenazadas, las intenciones son impuestas a los seres humanos por la opacidad de la figuración social en que se mueven, es decir, por la interdependencia no intencional de sus intenciones. Es justamente porque los individuos poseen un grado distinto de autonomía en diferentes situaciones, las cuales los presionan para comportarse de una determinada manera, por lo que se constituye el carácter ordenado de lo social y el desarrollo a largo plazo de los procesos sociales. Por ello, las dimensiones políticas y económicas, ejemplificadoras de contextos de competencia y monopolización, asumen en la tesis de Elias un papel importante en el desarrollo y dirección del «proceso civilizatorio».

La presión en situaciones de elección es normalmente sentida cuando la existencia social de las unidades en cuestión se encuentra amenazada -inicialmente, en el periodo del Bajo Medievo, bajo la forma indiferenciada de competencia política y económica; y más tarde, a partir del Renacimiento, cuando la monopolización de la violencia física se institucionaliza, y la competencia se encuadra ya dentro de un territorio pacificado, encontrando expresión en distintos rasgos de acciones y en distintos canales de movilidad social-. Con el paso del tiempo, la presión de la competencia «económico-política» en la Europa Occidental ha conducido a una diferenciación funcional creciente y a una integración social de interdependencias. Estos dos elementos han contribuido a la formación de instituciones de monopolización de la violencia física y a la emergencia de instituciones de centralización de los medios de producción, o empresas económicas, que han sido significativas en la continuación del desarrollo occidental. Estas dos dimensiones del desarrollo societal han podido ser coordinadas de tal forma que las dos se interpenetraron recíproca y diacrónicamente a lo largo de la historia.

La construcción del Estado Moderno constituye un ejemplo apropiado para ilustrar el concepto eliasiano de monopolización. Si bien esa construcción pasa por el afianzamiento de tendencias monopolizadoras, éstas son indisociables, como hemos visto, de dos fenóme- 
nos tales como la competencia y el poder. El Estado Moderno, consolidado en los siglos XVI y XVII, como institución monopolizadora de la tributación fiscal, la función administrativa y el uso legítimo de la fuerza física, encuentra su origen en una multiplicidad de pequeñas unidades políticas vinculadas entre sí por la competencia territorial a lo largo y ancho de la Edad Media. Semejante proceso de competencia conlleva que unas triunfen sobre otras, consiguiendo con estos triunfos oportunidades crecientes de poder sobre sus competidoras. Tales triunfos reducen progresivamente el número de unidades políticas participantes, al tiempo que las victoriosas traducen su poder en una mayor fuerza militar, en una mayor eficacia organizativa y en un mayor control sobre las fronteras y el interior de sus territorios. El desequilibrio en la cuota de poder es máximo cuando una de estas unidades políticas —encabezada por una casa real, por una estirpe nobiliaria - asume la totalidad de los recursos para controlar definitivamente un territorio en detrimento de aquellas cuantas competían. Aquí se dan las condiciones para que surja el Estado como entidad que, tras una intensa competencia, asume el monopolio de lo militar, lo fiscal y lo territorial, acrecentándose así sus oportunidades de poder sobre nuevos adversarios; en este caso, nuevos Estados.

Así, es la relativa similitud y diversidad de constreñimientos inherentes a las figuraciones en competencia la que permite avistar patrones recurrentes y un orden secuencial a la estructura de los procesos a largo plazo. La variabilidad de la dirección de la competencia es dependiente del aumento o disminución de la monopolización y centralización de los recursos y de los diferenciales de poder. En este sentido, los términos competencia y poder están relacionados. El concepto de diferencial de poder permite describir las tensiones inmanentes y el juego de fuerzas en las figuraciones en competencia de una forma específica. Obsérvese aquí la manera cómo el poder impulsa y condiciona la formación de una estructura no intencional en los procesos sociales a largo plazo.

En resumen, en esta obra, Elias asume la tesis de que el cambio histórico civilizatorio occidental surge de procesos no planeados aunque indudablemente ordenados. Esta tendencia civilizatoria es la consecuencia final de la evolución de ciertos procesos con una génesis no planeada, a saber: la creciente diferenciación social anclada en la división del trabajo, la formación del Estado Moderno y su concentración de los medios de control de la violencia y de tributación fiscal, y el aumento de la interdependencia entre los individuos que conduce a un equilibrio de los diferenciales de poder entre varios grupos. A la vez que las cadenas de interdependencia se tornan mayores y más complejas y que el Estado centraliza en sí el poder de pacificación social, las formas de comportamiento más violentas y hostiles se aminoran, contribuyendo a la solidificación de conductas más refinadas y civilizadas. El hilo conductor subyacente a las relaciones humanas del proceso civilizatorio occidental coge su dinámica en la oscilación de formas distintas de competencia y de mono- 
polización del poder. Esta tensión procesual construye un camino que nadie ha planeado, al que todos y nadie han contribuido.

Elias dedicó líneas y líneas de sociología a estudiar este proceso. Con algunas menos, aunque en verso, trató de ilustrar su devenir.

«born from a storm of disorder nomads of time without tiding in a void without border riding the storm"

Norbert Elias

\section{PONDERACIÓN FINAL}

A lo largo de este trabajo se ha procurado efectuar una incursión crítica en la manera en la que la teoría de la figuración eliasiana procura superar, a través de la metáfora de los modelos de juego, diversas líneas dualistas ancladas en teorías sociológicas clásicas. Pese a las fragilidades apuntadas, la empresa analítica del autor constituye un significativo y precursor avance de síntesis sociológica, sobre todo si consideramos el carácter autodidacta de su trayectoria profesional y la fecha en la que fue escrita su opus magnum, en 1939, mucho antes de cualquier intento analítico con este objetivo. Una de las ideas centrales que Elias introduce con la intención de superar este dualismo es la de que la sociedad está constituida por individuos interdependientes, cuyas acciones intencionales producen consecuencias inicialmente no pretendidas, reflejando esto la dinámica procesual de la realidad social. Las consecuencias no intencionales de las acciones humanas son así el fruto, no planeado pero ordenado, de las figuraciones sociales.

Con todo, pese a la descripción abstracta ilustrativa de un nivel sincrónico en los modelos de juego, y de otra más diacrónica —el proceso de la civilización- que cumple con rigor los objetivos propuestos por el autor, una de las críticas que procuramos aquí subrayar es la ambigua operatividad del término figuración en el análisis de la realidad social. De hecho, este concepto posee más un valor descriptivo que explicativo en la observación de las redes humanas, dado que intenta teorizar un «relacionismo procesual», atravesado por «diferenciales de poder», de carácter generalista e inherente a la propia relación social; concepto que procura explicar todo pero apenas lo logra con superficialidad. Nuestra tesis aquí es que Elias, en un nivel teórico, no consigue profundizar en el concepto de figuración, ni relacionarlo debidamente con otros elementos subjetivos (deseos, emociones, conciencia) que influyen explícitamente en el poder a la hora de actuar. 
En este sentido, una de las posibles líneas temáticas a desarrollar en un ensayo futuro sería la de observar cómo el concepto de figuración se puede extender, en articulación con otros conceptos de cariz interaccionista, hacia una mayor y más clara operatividad analítica. Otra propuesta temática distinta podría ser el análisis de la manera en la que el autor refleja en sus escritos poéticos matices de su programa sociológico «no intencionado ni planeado» 65 .

Pese a las críticas incluidas en esta ponderación final, Elias nítidamente logra en su obra una ilustración innovadora de un cambio civilizatorio, el occidental, que nos pertenece a todos, aunque sin intención inicial.

\section{ABSTRACT}

The aim of this paper is to analyse the theorectical centrality of a classic subject in sociology — «the unintended consequences of social action» (UCA) — through an exploration of the work of Norbert Elias. First of all, the paper tries to delineate the analytical bases of the UCA, having as a starting point the critical revision made by Ramón Ramos, who tries to revitalise its operational character in contemporary sociology. Secondly, in line with Ramos's proposal, the way Elias develops the task of focusing the subject of sociology through the overcoming of certain traditional dualisms —individual/society, action/structure, micro/macro_-, introducing at the center of his analysis the concept of «figuration», as a synthetical promise in social theory. This concept integrates two analytical levels —intended and unintended structures — that are frecuently differentiated from one another. Elias, in the figurational process postulates the existance of: a) unintended interdependencies between intentional actions; b) these unintended interdependencies remain over the intentional actions of the individuals, when one analyses social processes. In this way, intentions and individuals are made by unintended interdependencies in the figurational process. Finally, the theorectical strengths and weaknesses of Elias's proposal will be discussed in view of his persistant attempt of distancing his views from holism and from methodological individualism.

65 Una propuesta semejante a ésta es la de J. M. González García, quien sugiere partir de la interpretación de la poesía de Elias para analizar rasgos distintivos de su vida y obra. Vid. J. M. González García, «Norbert Elias: la literatura y la sociología en el proceso de la civilización», REIS, 65, 1994, pp. 55-77. 